\title{
Determination of CP and CPT violation parameters in the neutral kaon system using the Bell-Steinberger relation and WA data
}

Mario Antonelli*

$L N F-I N F N$

E-mail: mario.antonellielnf.infn.it

I present an improved determination of the $C P$ and $C P T$ violation parameters $\operatorname{Re}(\varepsilon)$ and $\operatorname{Im}(\delta)$ based on the unitarity condition (Bell-Steinberger relation) and on world average results from kaon experiments. We find $\operatorname{Re}(\varepsilon)=(161.1 \pm 0.5) \times 10^{-5}$ and $\operatorname{Im}(\delta)=(-0.7 \pm 1.4) \times 10^{-5}$, consistent with no $C P T$ violation. Assuming no CPT violation in decays one can derive: $\left|m_{K^{0}}-m_{\bar{K}^{0}}\right|<4 \times 10^{-19} \mathrm{GeV}$ at $95 \%$ C.L.

The 7th International Workshop on Chiral Dynamics, August 6 -10, 2012

Jefferson Lab, Newport News, Virginia, USA

${ }^{*}$ Speaker. 
The three discrete symmetries of quantum mechanics, charge conjugation $(C)$, parity $(P)$ and time reversal $(T)$, are known to be violated in nature, both singly and in bilinear combinations. Only $C P T$ appears to be an exact symmetry of nature. Exact $C P T$ invariance holds in quantum field theory, which assumes Lorentz invariance (flat space), locality and unitarity [1]. Testing the validity of $C P T$ invariance therefore probes the most fundamental assumptions of our present understanding of particles and their interactions. These hypotheses are likely to be violated at very high energy scales, where quantum effects of the gravitational interaction cannot be ignored [2]. On the other hand, since we still lack a consistent theory of quantum gravity, it is hard to predict at which level violation of $C P T$ invariance might become experimentally observable.

The neutral kaon system offers unique possibilities for the study of $C P T$ invariance. From the requirement of unitarity, Bell and Steinberger have derived a relation, the so-called BellSteinberger relation [3]. The Bell-Steinberger relation relates a possible violation of $C P T$ invariance $\left(m_{K^{0}} \neq m_{\bar{K}^{0}}\right.$ and/or $\left.\Gamma_{K^{0}} \neq \Gamma_{\bar{K}^{0}}\right)$ in the time-evolution of the $K^{0}-\bar{K}^{0}$ system to the observable $C P$-violating interference of $K_{L}$ and $K_{S}$ decays into the same final state $f$. Strictly speaking, evidence of $C P T$ violation found via the Bell-Steinberger relation could just be a failure of the unitarity assumption. However, unitarity is also one of the main hypotheses of the $C P T$ theorem; thus the Bell-Steinberger relation allows a test of the basic assumptions of quantum field theories.

In this work we use recent results from the Kaon experiments to improve the determination of the phenomenological $C P$ - and $C P T$-violating parameters $\operatorname{Re}(\varepsilon)$ and $\operatorname{Im}(\delta)$ by means of the BSR.

Strangeness oscillation in $K^{0}-\bar{K}^{0}$ system, described by the equation

$$
i \frac{d}{d t}\left[\begin{array}{c}
K^{0} \\
\bar{K}^{0}
\end{array}\right]=[M-i \Gamma / 2]\left[\begin{array}{c}
K^{0} \\
\bar{K}^{0}
\end{array}\right],
$$

where $M$ and $\Gamma$ are hermitian matrices (see PDG review[4], references [5][6], and KLOE paper[7] for notations and previous literature), allows a very accurate test of $C P T$ symmetry; indeed since $C P T$ requires $M_{11}=M_{22}$ and $\Gamma_{11}=\Gamma_{22}$, the mass and width eigenstates, $K_{S, L}$, have a $C P T$-violating piece, $\delta$, in addition to the usual $C P T$-conserving parameter $\varepsilon$ :

$$
\begin{gathered}
K_{S, L}=\frac{1}{\sqrt{2\left(1+\left|\varepsilon_{S, L}\right|^{2}\right)}}\left[\left(1+\varepsilon_{S, L}\right) K^{0}+\left(1-\varepsilon_{S, L}\right) \bar{K}^{0}\right] \\
\varepsilon_{S, L}=\frac{-i \operatorname{Im}\left(M_{12}\right)-\frac{1}{2} \operatorname{Im}\left(\Gamma_{12}\right) \mp \frac{1}{2}\left[M_{11}-M_{22}-\frac{i}{2}\left(\Gamma_{11}-\Gamma_{22}\right)\right]}{m_{L}-m_{S}+i\left(\Gamma_{S}-\Gamma_{L}\right) / 2} \\
\equiv \varepsilon \pm \delta .
\end{gathered}
$$

Using the phase convention $\operatorname{Im}\left(\Gamma_{12}\right)=0$, we determine the phase of $\varepsilon$ to be $\varphi_{S W} \equiv \arctan \frac{2\left(m_{L}-m_{S}\right)}{\Gamma_{S}-\Gamma_{L}}$. Imposing unitarity to an arbitrary combination of $K^{0}$ and $\bar{K}^{0}$ wave functions, we obtain the BellSteinberger relation[8] connecting $C P$ and $C P T$ violation in the mass matrix to $C P$ and $C P T$ violation in the decay; in fact, neglecting $\mathscr{O}(\varepsilon)$ corrections to the coefficient of the $C P T$-violating parameter, $\delta$, we can write[7]

$$
\left[\frac{\Gamma_{S}+\Gamma_{L}}{\Gamma_{S}-\Gamma_{L}}+i \tan \phi_{\mathrm{SW}}\right]\left[\frac{\operatorname{Re}(\varepsilon)}{1+|\varepsilon|^{2}}-i \operatorname{Im}(\delta)\right]=\frac{1}{\Gamma_{S}-\Gamma_{L}} \sum_{f} A_{L}(f) A_{S}^{*}(f)
$$


where $A_{L, S}(f) \equiv A\left(K_{L, S} \rightarrow f\right)$. We stress that this relation is phase-convention-independent. The advantage of the neutral kaon system is that only a few decay modes give significant contributions to the r.h.s. in Bell-Steinberger relation; in fact, defining for the hadronic modes

$$
\begin{aligned}
\alpha_{i} & \equiv \frac{1}{\Gamma_{S}}\left\langle\mathscr{A}_{L}(i) \mathscr{A}_{S}^{*}(i)\right\rangle=\eta_{i} \mathscr{B}\left(K_{S} \rightarrow i\right), \\
i & =\pi^{0} \pi^{0}, \pi^{+} \pi^{-}(\gamma), 3 \pi^{0}, \pi^{0} \pi^{+} \pi^{-}(\gamma),
\end{aligned}
$$

the recent data from CPLEAR, KLOE, KTeV, and NA48 have led to the following determinations the analysis described in Ref [7] has been updated by using the recent measurements of $K_{L}$ branching ratios from $\mathrm{KTeV}$ [9], $\mathrm{NA48[10]} \mathrm{and} \mathrm{the} \mathrm{recent} \mathrm{result} \mathrm{obtained} \mathrm{by} \mathrm{KTeV}$ on the $\mathrm{CP}$ phases[?].

$$
\begin{gathered}
\alpha_{\pi^{+} \pi^{-}}=((1.112 \pm 0.010)+i(1.061 \pm 0.010)) \times 10^{-3}, \\
\alpha_{\pi^{0} \pi^{0}}=((0.493 \pm 0.005)+i(0.471 \pm 0.005)) \times 10^{-3}, \\
\alpha_{\pi^{+} \pi^{-} \pi^{0}}=((0 \pm 2)+i(0 \pm 2)) \times 10^{-6} \\
\left|\alpha_{\pi^{0} \pi^{0} \pi^{0}}\right|<7 \times 10^{-6} \text { at } 95 \% \mathrm{CL}
\end{gathered}
$$

The semileptonic contribution to the right-handed side of Bell-Steinberger relation requires the determination of several observables: we define[5][6]

$$
\begin{gathered}
\mathscr{A}\left(K^{0} \rightarrow \pi^{-} l^{+} v\right)=\mathscr{A}_{0}(1-y), \\
\mathscr{A}\left(K^{0} \rightarrow \pi^{+} l^{-} v\right)=\mathscr{A}_{0}^{*}\left(1+y^{*}\right)\left(x_{+}-x_{-}\right)^{*}, \\
\mathscr{A}\left(\bar{K}^{0} \rightarrow \pi^{+} l^{-} v\right)=\mathscr{A}_{0}^{*}\left(1+y^{*}\right), \\
\mathscr{A}\left(\bar{K}^{0} \rightarrow \pi^{-} l^{+} v\right)=\mathscr{A}_{0}(1-y)\left(x_{+}+x_{-}\right)
\end{gathered}
$$

where $x_{+}\left(x_{-}\right)$describes the violation of the $\Delta S=\Delta Q$ rule in $C P T$-conserving (violating) decay amplitudes, and $y$ parametrizes $C P T$ violation for $\Delta S=\Delta Q$ transitions. Taking advantage of their tagged $K^{0}\left(\bar{K}^{0}\right)$ beams, CPLEAR has measured $\operatorname{Im}\left(x_{+}\right), \operatorname{Re}\left(x_{-}\right), \operatorname{Im}(\delta)$, and $\operatorname{Re}(\delta)[13]$. These determinations have been improved in $\operatorname{Ref}$ [7] by including the information $A_{S}-A_{L}=4[\operatorname{Re}(\delta)+$ $\operatorname{Re}\left(x_{-}\right)$], where $A_{L, S}$ are the $K_{L}$ and $K_{S}$ semileptonic charge asymmetries, respectively, from the PDG[14] and KLOE[15]. Here we are also including the $T$-violating asymmetry measurement from CPLEAR[16].

The value $A_{S}+A_{L}$ in Table 1 can be directely included in the semileptonic contributions to the Bell Steinberger relations in Bell-Steinberger relation

$$
\begin{gathered}
\sum_{\pi \ell v}\left\langle\mathscr{A}_{L}(\pi \ell v) \mathscr{A}_{S}^{*}(\pi \ell v)\right\rangle=2 \Gamma\left(K_{L} \rightarrow \pi \ell v\right)\left(\operatorname{Re}(\varepsilon)-\operatorname{Re}(y)-i\left(\operatorname{Im}\left(x_{+}\right)+\operatorname{Im}(\delta)\right)\right) \\
=2 \Gamma\left(K_{L} \rightarrow \pi \ell v\right)\left(\left(A_{S}+A_{L}\right) / 4-i\left(\operatorname{Im}\left(x_{+}\right)+\operatorname{Im}(\delta)\right)\right) \\
\alpha_{\pi \ell v} \equiv \frac{1}{\Gamma_{S}} \sum_{\pi \ell v}\left\langle\mathscr{A}_{L}(\pi \ell v) \mathscr{A}_{S}^{*}(\pi \ell v)\right\rangle+2 i \frac{\tau_{K_{S}}}{\tau_{K_{L}}} \mathscr{B}\left(K_{L} \rightarrow \pi \ell v\right) \operatorname{Im}(\delta)
\end{gathered}
$$

we find:

$$
\alpha_{\pi \ell v}=((-0.2 \pm 0.5)+i(0.1 \pm 0.5)) \times 10^{-5}
$$




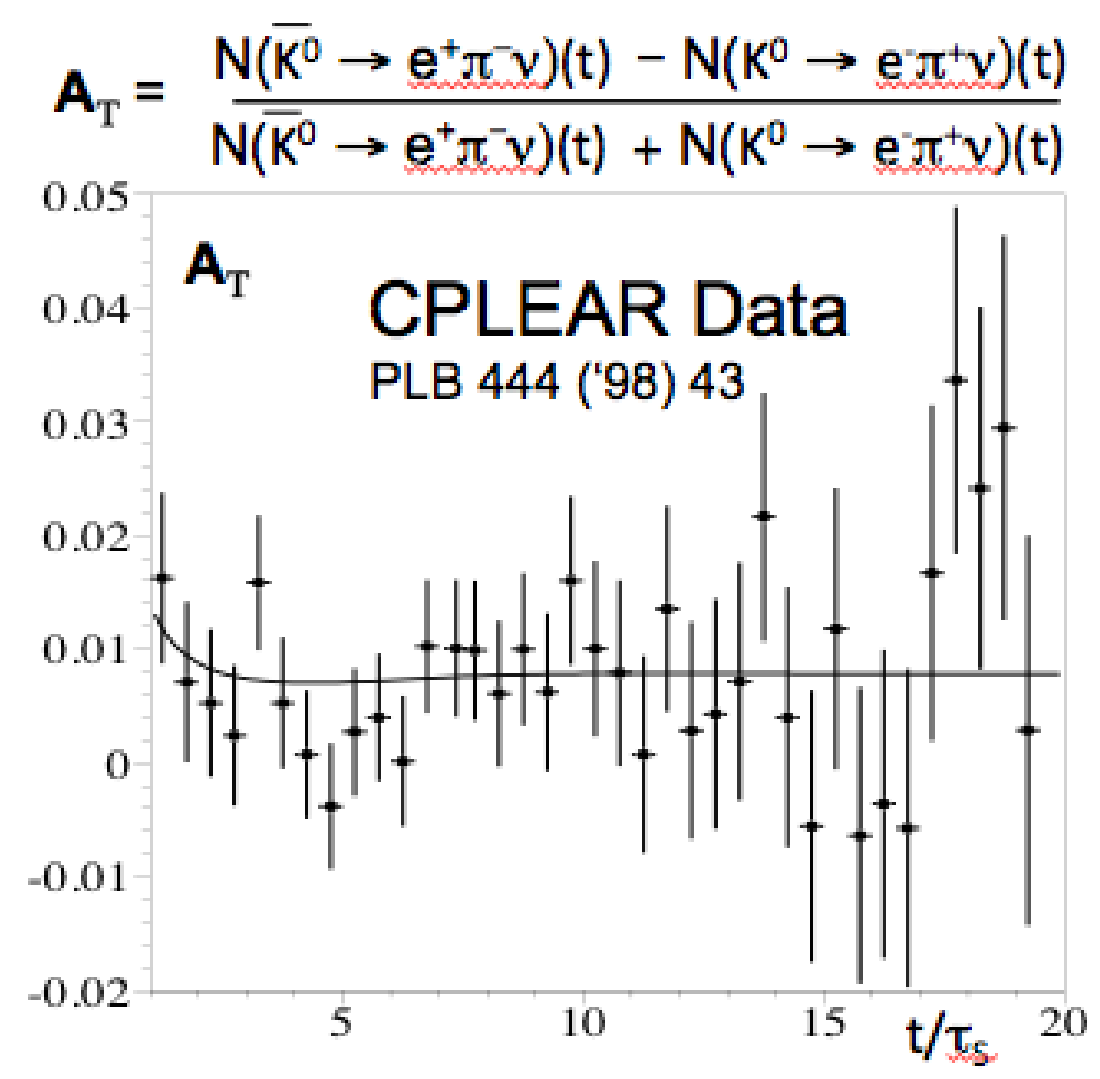

Figure 1: CPLEAR $A_{T}$ measurement and our fit result.

\begin{tabular}{l|c|ccccc}
\hline \hline & value & \multicolumn{5}{|c}{ Correlation coefficients } \\
\hline $\operatorname{Re}(\boldsymbol{\delta})$ & $(3.0 \pm 2.3) \times 10^{-4}$ & 1 & & & & \\
$\operatorname{Im}(\boldsymbol{\delta})$ & $(-0.66 \pm 0.65) \times 10^{-2}$ & -0.21 & 1 & & & \\
$\operatorname{Re}\left(x_{-}\right)$ & $(-0.30 \pm 0.21) \times 10^{-2}$ & -0.21 & -0.60 & 1 & & \\
$\operatorname{Im}\left(x_{+}\right)$ & $(0.02 \pm 0.22) \times 10^{-2}$ & -0.38 & -0.14 & 0.47 & 1 & \\
$A_{S}+A_{L}$ & $(-0.40 \pm 0.83) \times 10^{-2}$ & -0.10 & -0.63 & 0.99 & 0.43 & 1
\end{tabular}

Table 1: Values, errors, and correlation coefficients for $\operatorname{Re}(\delta), \operatorname{Im}(\delta), \operatorname{Re}\left(x_{-}\right), \operatorname{Im}\left(x_{+}\right)$, and $A_{S}+A_{L}$ obtained from a combined fit, including KLOE [7] and CPLEAR [16].

Inserting the values of the $\alpha$ parameters into Bell-Steinberger relation, we find

$$
\begin{aligned}
& \operatorname{Re}(\varepsilon)=(161.1 \pm 0.5) \times 10^{-5} \\
& \operatorname{Im}(\delta)=(-0.7 \pm 1.4) \times 10^{-5}
\end{aligned}
$$

The complete information is given in Table 2 .

Now the agreement with $C P T$ conservation, $\operatorname{Im}(\delta)=\operatorname{Re}(\delta)=\operatorname{Re}\left(x_{-}\right)=0$, is at $20 \%$ C.L. 


\begin{tabular}{l|c|cccc} 
& value & \multicolumn{5}{|c}{ Correlation coefficients } \\
\hline $\operatorname{Re}(\varepsilon)$ & $(161.1 \pm 0.5) \times 10^{-5}$ & +1 & & & \\
$\operatorname{Im}(\delta)$ & $(-0.7 \pm 1.4) \times 10^{-5}$ & +0.09 & 1 & & \\
$\operatorname{Re}(\delta)$ & $(2.4 \pm 2.3) \times 10^{-4}$ & +0.08 & -0.12 & 1 & \\
$\operatorname{Re}\left(x_{-}\right)$ & $(-4.1 \pm 1.7) \times 10^{-3}$ & +0.14 & 0.22 & -0.43 & 1
\end{tabular}

Table 2: Summary of results: values, errors, and correlation coefficients for $\operatorname{Re}(\varepsilon), \operatorname{Im}(\delta), \operatorname{Re}(\delta)$, and $\operatorname{Re}\left(x_{-}\right)$.

The allowed region in the $\operatorname{Re}(\varepsilon)-\operatorname{Im}(\delta)$ plane at $68 \% \mathrm{CL}$ and $95 \%$ C.L. is shown in the top panel of Fig 2.
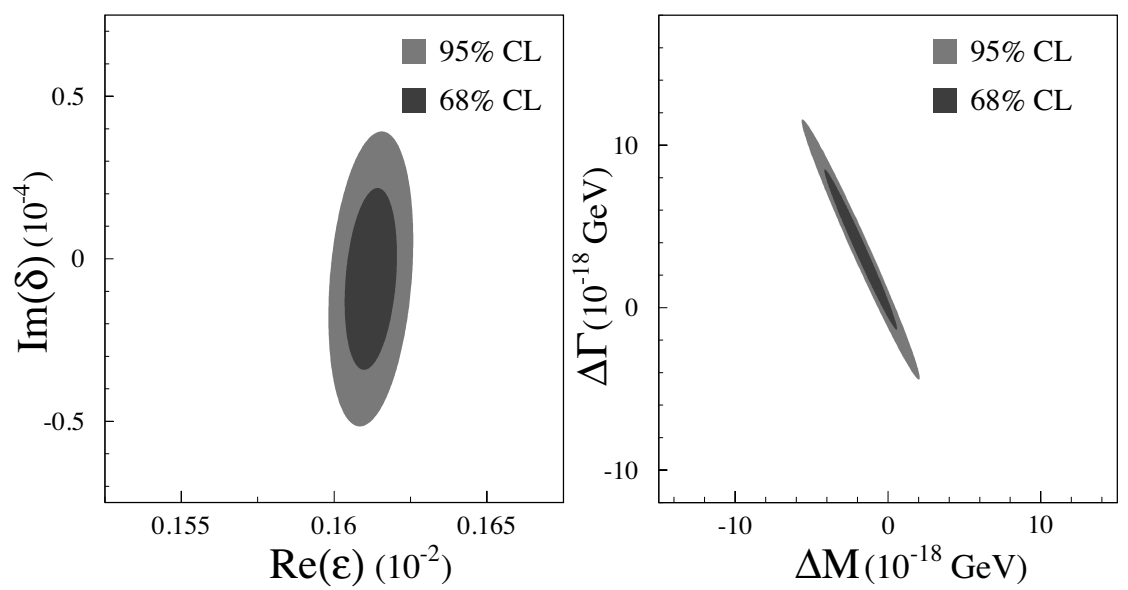

Figure 2: Left: allowed region at $68 \%$ and $95 \% \mathrm{CL}$ in the $\operatorname{Re}(\varepsilon), \operatorname{Im}(\delta)$ plane. Right: allowed region at $68 \%$ and $95 \%$ CL in the $\Delta M, \Delta \Gamma$ plane.

The process giving the largest contribution to the size of the allowed region is $K_{L} \rightarrow \pi^{+} \pi^{-}$, through the uncertainty on $\phi_{+-}$.

The limits on $\operatorname{Im}(\delta)$ and $\operatorname{Re}(\delta)$ can be used to constrain the $K^{0}-\bar{K}^{0}$ mass and width difference

$$
\delta=\frac{i\left(m_{K^{0}}-m_{\bar{K}^{0}}\right)+\frac{1}{2}\left(\Gamma_{K^{0}}-\Gamma_{\bar{K}^{0}}\right)}{\Gamma_{S}-\Gamma_{L}} \cos \phi_{S W} e^{i \phi_{S W}}[1+\mathscr{O}(\varepsilon)] .
$$

The allowed region in the $\Delta M=\left(m_{K^{0}}-m_{\bar{K}^{0}}\right), \Delta \Gamma=\left(\Gamma_{K^{0}}-\Gamma_{\bar{K}^{0}}\right)$ plane is shown in the bottom panel of Fig 2.

As a result, we improve on the previous limits (see for instance, P. Bloch in Ref. [14]) and in the limit $\Gamma_{K^{0}}-\Gamma_{\bar{K}^{0}}=0$ we obtain

$$
-4 \times 10^{-19} \mathrm{GeV}<m_{K^{0}}-m_{\bar{K}^{0}}<4 \times 10^{-19} \mathrm{GeV} \text { at } 95 \% \text { C.L. }
$$

\section{References}

[1] G. Lüders, Ann. Phys. 2 (1957) 1, reprinted in Ann. Phys. 281 (2000) 1004. 
[2] See e.g. J. Bernabeu, J. Ellis, N. E. Mavromatos, D. V. Nanopoulos and J. Papavassiliou, hep-ph/0607322; V.A. Kostelecky and R. Lehnert, Phys. Rev. D632001065008; and references therein.

[3] J.S. Bell and J. Steinberger, Proceedings Oxford Int. Conf. on Elementary Particles (1965).

[4] W. M. Yao et al. "Review of particle physics,” J. Phys. G 33 (2006) 1

[5] L. Maiani, “ $C P$ And $C P T$ Violation In Neutral Kaon Decays,” L. Maiani, G. Pancheri, and N. Paver, The Second Daphne Physics Handbook, Vol. 1, 2

[6] G. D’Ambrosio, G. Isidori, and A. Pugliese, “ $C P$ and $C P T$ mesurements at DAФNE,” L. Maiani, G. Pancheri, and N. Paver, The Second Daphne Physics Handbook, Vol. 1, 2

[7] F. Ambrosino et al, [KLOE Collaboration], "Determination of CP and CPT violation parameters in the neutral kaon system using the Bell-Steinberger relation and data from the KLOE experiment," JHEP0612,011(2006).

[8] J. S. Bell and J. Steinberger, “Weak Interactions Of Kaons,” In Wolfenstein, L. (ed.): CP violation, 42-57. (In Oxford International Symposium Conference on Elementary Particles, September 1965,195-208, 221-222). (See Book Index)

[9] T. Alexopoulos et al, [KTeV Collaboration], "A determination of the CPT violation parameter Re(delta) from the semileptonic decay of strangeness-tagged neutral kaons,” Phys. Rev. D70,092006(1998)

[10] A. Lai et al, [NA48 Collaboration], Phys. Lett. B 645, 26 (2007)

[11] A. Lai et al, [NA48 Collaboration], Phys. Lett. B 602, 41 (2004)

[12] A. Angelopoulos et al, [CPLEAR Collaboration], "Physics at CPLEAR,” Phys. Rept. 374 (2003) 165

[13] A. Angelopoulos et al, [CPLEAR Collaboration], "A determination of the CPT violation parameter Re(delta) from the semileptonic decay of strangeness-tagged neutral kaons,” Phys. Lett. B 444, 52 (1998)

[14] W. M. Yao et al, [Particle Data Group], “Review of particle physics,” J. Phys. G 33 (2006) 1

[15] F. Ambrosino et al, [KLOE Collaboration], "Measurement of the branching fraction and charge asymmetry for the decay $K(S) \rightarrow$ pi e nu with the KLOE detector,” Phys. Lett. B 636 (2006) 173 [arXiv:hep-ex/0601026]

[16] P. Bloch, M. Fidecaro, private communication of the data in a finer binning format; A. Angelopoulos et al, [CPLEAR Collaboration], Phys. Lett. B 444 (1998) 43

[17] E. Abouzaid et al. [KTeV Collaboration], "Precise Measurements of Direct CP Violation, CPT Symmetry, and Other Parameters in the Neutral Kaon System,"

Phys. Rev. D 83, 092001 (2011) [arXiv:1011.0127 [hep-ex] ]. 